\title{
Besov Spaces Induced by Doubling Weights
}

\section{Atte Reijonen ${ }^{1}$}

Received: 26 June 2019 / Accepted: 8 November 2019 / Published online: 22 January 2020

(c) The Author(s) 2020

\section{Abstract}

Let $1 \leqslant p<\infty, 0<q<\infty$, and $v$ be a two-sided doubling weight satisfying

$$
\sup _{0 \leqslant r<1} \frac{(1-r)^{q}}{\int_{r}^{1} v(t) d t} \int_{0}^{r} \frac{v(s)}{(1-s)^{q}} d s<\infty .
$$

The weighted Besov space $\mathcal{B}_{v}^{p, q}$ consists of those $f \in H^{p}$ such that

$$
\int_{0}^{1}\left(\int_{0}^{2 \pi}\left|f^{\prime}\left(r e^{i \theta}\right)\right|^{p} d \theta\right)^{q / p} v(r) d r<\infty .
$$

Our main result gives a characterization for $f \in \mathcal{B}_{v}^{p, q}$ depending only on $|f|, p, q$, and $v$. As a consequence of the main result and inner-outer factorization, we obtain several interesting by-products. For instance, we show the following modification of a classical factorization by $\mathrm{F}$. and R. Nevanlinna: If $f \in \mathcal{B}_{v}^{p, q}$, then there exist $f_{1}, f_{2} \in \mathcal{B}_{v}^{p, q} \cap H^{\infty}$ such that $f=f_{1} / f_{2}$. Moreover, we give a sufficient and necessary condition guaranteeing that the product of $f \in H^{p}$ and an inner function belongs to $\mathcal{B}_{v}^{p, q}$. Applying this result, we make some observations on zero sets of $\mathcal{B}_{v}^{p, p}$.

Keywords Doubling weight $\cdot$ Besov space $\cdot$ Hardy space $\cdot$ Inner-outer factorization · Mixed norm space $\cdot$ Zero set

Mathematics Subject Classification Primary: 30H10 $\cdot 30 \mathrm{H} 25$

Communicated by G. Kerkyacharian.

This research was supported in part by Academy of Finland Project No. 286877 and Finnish Cultural Foundation.

\footnotetext{
$凶 \quad$ Atte Reijonen

atte.reijonen@uef.fi

1 University of Eastern Finland, P.O. Box 111, 80101 Joensuu, Finland
} 


\section{Introduction and Characterizations}

Let $\mathbb{D}$ be the open unit disc of the complex plane $\mathbb{C}$ and $\mathbb{T}$ be the boundary of $\mathbb{D}$. The set of all analytic functions in $\mathbb{D}$ is denoted by $\mathcal{H}(\mathbb{D})$. For $0<p<\infty$, the Hardy space $H^{p}$ consists of those $f \in \mathcal{H}(\mathbb{D})$ such that

$$
\|f\|_{H^{p}}=\sup _{0 \leqslant r<1} M_{p}(r, f)<\infty
$$

where

$$
M_{p}(r, f)=\left(\frac{1}{2 \pi} \int_{0}^{2 \pi}\left|f\left(r e^{i \theta}\right)\right|^{p} d \theta\right)^{1 / p}
$$

The Hardy space $H^{\infty}$ is the set of all bounded functions in $\mathcal{H}(\mathbb{D})$. Moreover, we recall that a measurable function $f$ on $\mathbb{T}$ belongs to $L^{p}(\mathbb{T})$ for some $p \in(0, \infty)$ if

$$
\|f\|_{L^{p}}^{p}=\frac{1}{2 \pi} \int_{0}^{2 \pi}\left|f\left(e^{i \theta}\right)\right|^{p} d \theta<\infty .
$$

Alternatively, the Hardy space $H^{p}$ for $0<p<\infty$ can be characterized as follows: $f \in H^{p}$ if and only if $f \in \mathcal{H}(\mathbb{D})$, the nontangential limit $f\left(e^{i \theta}\right)$ exists almost everywhere on $\mathbb{T}$, and $f\left(e^{i \theta}\right) \in L^{p}(\mathbb{T})$. In particular, $\|f\|_{H^{p}}=\|f\|_{L^{p}}$ for $0<p<\infty$ and $f \in H^{p}$. This is due to Hardy's convexity and the mean convergence theorems. These results and much more can be found in the classic book [8] by P. Duren.

A function $v: \mathbb{D} \rightarrow[0, \infty)$ is called a (radial) weight if it is integrable over $\mathbb{D}$ and $v(z)=v(|z|)$ for all $z \in \mathbb{D}$. For $0<p, q<\infty$, and a weight $v$, the weighted mixed norm space $A_{v}^{p, q}$ consists of those $f \in \mathcal{H}(\mathbb{D})$ such that

$$
\|f\|_{A_{v}^{p, q}}^{q}=\int_{0}^{1} M_{p}^{q}(r, f) v(r) d r<\infty .
$$

If $v(z)=(1-|z|)^{\alpha}$ for $-1<\alpha<\infty$, then the notation $A_{\alpha}^{p, q}$ is used for $A_{v}^{p, q}$. In this paper, we study class $\mathcal{D}$ of so-called two-sided doubling weights, which originates from the work of J. A. Peláez and J. Rättyä [19,20]. For the definition of $\mathcal{D}$ we have to define two wider classes. For a weight $v$, set

$$
\widehat{v}(z)=\widehat{v}(|z|)=\int_{|z|}^{1} v(s) d s, \quad z \in \mathbb{D} .
$$

If a weight $v$ satisfies the condition $\widehat{v}(r) \leqslant C \widehat{v}\left(\frac{1+r}{2}\right)$ for all $0 \leqslant r<1$ and some $C=$ $C(v)>0$, then we write $v \in \widehat{\mathcal{D}}$. Correspondingly, $v \in \check{\mathcal{D}}$ if there exist $K=K(v)>1$ and $C=C(v)>1$ such that

$$
\widehat{v}(r) \geqslant C \widehat{v}\left(1-\frac{1-r}{K}\right), \quad 0 \leqslant r<1 .
$$


Class $\mathcal{D}$ is the intersection of $\widehat{\mathcal{D}}$ and $\check{\mathcal{D}}$. In addition, we define the following subclass of $\widehat{\mathcal{D}}: v \in \widehat{\mathcal{D}}_{p}$ for some $p \in(0, \infty)$ if the condition

$$
\widehat{\mathcal{D}}_{p}(v)=\sup _{0 \leqslant r<1} \frac{(1-r)^{p}}{\widehat{v}(r)} \int_{0}^{r} \frac{v(s)}{(1-s)^{p}} d s<\infty
$$

is satisfied. As a concrete example, we mention that $\nu_{1}(z)=(1-|z|)^{\alpha}$ and $\nu_{2}(z)=(1-$ $|z|)^{\alpha}\left(\log \frac{e}{1-|z|}\right)^{\beta}$ for any $\beta \in \mathbb{R}$ belonging to $\mathcal{D} \cap \widehat{\mathcal{D}}_{p}$ if and only if $-1<\alpha<p-1$. Additional information about weights can be found in [18-20]. Some basic properties are recalled also in Sect. 2.

Define the weighted Besov space $\mathcal{B}_{v}^{p, q}$ by $\mathcal{B}_{v}^{p, q}=\left\{f: f^{\prime} \in A_{v}^{p, q}\right\} \cap H^{p}$. For $-1<\alpha<\infty$ and $v(z)=(1-|z|)^{\alpha}$, the notation $\mathcal{B}_{\alpha}^{p, q}$ is used for $\mathcal{B}_{v}^{p, q}$. The space $\mathcal{B}_{v}^{p, q}$ is the main research objective of this paper. Hence it is worth pointing out that the definition is rational, which means that $H^{p}$ is not a subset of $\left\{f: f^{\prime} \in A_{v}^{p, q}\right\}$ in general, or conversely. The family of Blaschke products offers examples for the case where $f \in H^{\infty}$ and $f^{\prime} \notin A_{v}^{p, q}$; see for instance [24]. Moreover, it would be natural that certain lacunary series $g$ lie out of $H^{p}$, while $g^{\prime} \in A_{v}^{p, q}$. Arguments for these kinds of examples can be found in M. Pavlović's book [16], which contains numerous important observations on the topic of this paper. The existence of both examples, of course, depends on $p, q$, and $v$. In other words, under certain hypotheses for $p, q$, and $v$, an inclusion relation between $\left\{f: f^{\prime} \in A_{v}^{p, q}\right\}$ and $H^{p}$ might be valid. However, this is not the case in general.

For $0<p<\infty$ and $f \in L^{p}(\mathbb{T})$, the $L^{p}$ modulus of continuity $\omega_{p}(t, f)$ is defined by

$$
\omega_{p}(t, f)=\sup _{0<h<t}\left(\int_{0}^{2 \pi}\left|f\left(e^{i(\theta+h)}\right)-f\left(e^{i \theta}\right)\right|^{p} d \theta\right)^{1 / p}, \quad 0<t \leqslant 2 \pi .
$$

We interpret $\omega_{p}(t, f)=\omega_{p}(2 \pi, f)$ for $t>2 \pi$. It is a well-known fact that, for $0<p, q<\infty,-1<\alpha<q-1$, and $f \in H^{p}$, the derivative of $f$ belongs to $A_{\alpha}^{p, q}$ if and only if

$$
\int_{0}^{\infty} \frac{\omega_{p}(t, f)^{q}}{t^{q-\alpha}} d t<\infty
$$

This result originates from E. M. Stein's book [26, Chapter V, Section 5], and the complete version is a consequence of [17, Theorems 2.1 and 5.1] or [12, Theorem 1.2] by M. Pavlović and M. Jevtić. Our first theorem is a partial generalization of the result. Its proof uses some ideas from [8,21,22].

Theorem 1 Let $1 \leqslant p<\infty, 0<q<\infty$, and $v \in \mathcal{D}$. Then $v \in \widehat{\mathcal{D}}_{q}$ if and only if there exists a constant $C=C(p, q, v)>0$ such that

$$
\int_{1 / 2}^{1} \omega_{p}(1-r, f)^{q} \frac{v(r)}{(1-r)^{q}} d r \leqslant C\left\|f^{\prime}\right\|_{A_{v}^{p, q}}^{q}
$$

for all $f \in H^{p}$. 
Note that (1.2) is valid also if $0<p, q<\infty, v$ is a weight, and there exists $\beta=\beta(q, v)<q-1$ such that $v(r) /(1-r)^{\beta}$ is increasing for $0 \leqslant r<1$. This is due to [17, Theorem 5.1] and its proof. Even though the result is valid also for $0<p<1$, Theorem 1 is more useful for our purposes. In particular, it is worth underlining that the hypothesis $1 \leqslant p<\infty$ in Theorems 2 and 3 below is natural.

Theorem 2 gives a practical estimate for $\left\|f^{\prime}\right\|_{A_{v}^{p, q}}^{q}+\|f\|_{H^{p}}^{q}$ when $f \in H^{p}$. As a by-product of its argument, we deduce that also the converse inequality of (1.2) holds if the norm $\|f\|_{H^{p}}^{q}$ is added into the right-hand side. Setting

$$
d \mu_{z}(\theta)=\frac{1-|z|^{2}}{\left|e^{i \theta}-z\right|^{2}} \frac{d \theta}{2 \pi}, \quad z \in \mathbb{D}, \quad 0 \leqslant \theta<2 \pi,
$$

Theorem 2 reads as follows.

Theorem 2 Let $1 \leqslant p<\infty, 0<q<\infty$, and $v \in \mathcal{D} \cap \widehat{\mathcal{D}}_{q}$. Then there exist positive constants $C_{1}$ and $C_{2}$ depending only on $p, q$, and $v$ such that

$$
\begin{aligned}
\left\|f^{\prime}\right\|_{A_{v}^{p, q}}^{q} & \leqslant \int_{0}^{1}\left(\int_{0}^{2 \pi}\left(\int_{0}^{2 \pi}\left|f\left(e^{i \theta}\right)-f\left(r e^{i t}\right)\right| d \mu_{r e^{i t}}(\theta)\right)^{p} d t\right)^{q / p} \frac{v(r)}{(1-r)^{q}} d r \\
& \leqslant C_{1}\left(\int_{0}^{1} \omega_{p}(1-s, f)^{q} \frac{v(s)}{(1-s)^{q}} d s+\|f\|_{H^{p}}^{q}\right) \\
& \leqslant C_{2}\left(\left\|f^{\prime}\right\|_{A_{v}^{p, q}}^{q}+\|f\|_{H^{p}}^{q}\right)
\end{aligned}
$$

for all $f \in H^{p}$.

By studying the classical weight $v(z)=(1-|z|)^{\alpha}$, where $-1<\alpha<q-1$, we obtain K. M. Dyakonov's [9, Proposition 2.2(a)] as a direct consequence of Theorem 2. Hence it does not come as a surprise that the proofs of [9, Theorem 2.1] and Theorem 2 have some similarities. Nonetheless, it is worth mentioning that the presence of general weights complicates the argument, and consequently, our proof is quite technical. Note also that Theorem 1 plays an essential role in the proof.

Our main result below gives a characterization for functions $f$ in $\mathcal{B}_{v}^{p, q}$ depending only $|f|, p, q$, and $v$. This result improves B. Bøe's [6, Theorem 1.1], which concentrates only on the case where $1 \leqslant p, q<\infty,-1<\alpha<q-1$, and $v(z)=(1-|z|)^{\alpha}$. It also generalizes the essential contents of [2, Proposition 2.4] and [9, Proposition 2.2(b)] made by A. Aleman and K. M. Dyakonov, respectively.

Theorem 3 Let $1 \leqslant p<\infty, 0<q<\infty$, and $v \in \mathcal{D} \cap \widehat{\mathcal{D}}_{q}$. Then there exist positive constants $C_{1}$ and $C_{2}$ depending only on $p, q$, and $v$ such that

$$
\left\|f^{\prime}\right\|_{A_{v}^{p, q}}^{q} \leqslant C_{1}\left(F_{1}(f)+F_{2}(f)\right) \leqslant C_{2}\left(\left\|f^{\prime}\right\|_{A_{v}^{p, q}}^{q}+\|f\|_{H^{p}}^{q}\right), \quad f \in H^{p},
$$

where

$$
F_{1}(f)=\int_{0}^{1}\left(\int_{0}^{2 \pi}\left(\int_{0}^{2 \pi}\left|f\left(e^{i \theta}\right)\right| d \mu_{r e^{i t}}(\theta)-\left|f\left(r e^{i t}\right)\right|\right)^{p} d t\right)^{q / p} \frac{v(r)}{(1-r)^{q}} d r
$$


and

$$
F_{2}(f)=\int_{0}^{1}\left(\int_{0}^{2 \pi}\left(\int_{0}^{2 \pi}|| f\left(e^{i \theta}\right)\left|-\int_{0}^{2 \pi}\right| f\left(e^{i s}\right)\left|d \mu_{r e^{i t}}(s)\right| d \mu_{r e^{i t}}(\theta)\right)^{p} d t\right)^{q / p} \frac{v(r)}{(1-r)^{q}} d r .
$$

Before we talk about the argument of Theorem 3, recall the inner-outer factorization. An inner function is a member of $H^{\infty}$ having unimodular radial limits almost everywhere on $\mathbb{T}$. For $0<p \leqslant \infty$, an outer function for $H^{p}$ takes the form

$$
O_{\phi}(z)=\exp \left(\frac{1}{2 \pi} \int_{0}^{2 \pi} \frac{e^{i \theta}+z}{e^{i \theta}-z} \log \phi\left(e^{i \theta}\right) d \theta\right), \quad z \in \mathbb{D}
$$

where $\phi$ is a non-negative function in $L^{p}(\mathbb{T})$ and $\log \phi \in L^{1}(\mathbb{T})$. The inner-outer factorization asserts that $f \in H^{p}$ can be represented as the product of an inner and outer function; see for instance [8, Theorem 2.8]. It is worth noting that the factorization is unique, and

$$
|f(\xi)|=\left|O_{\phi}(\xi)\right|=\phi(\xi)
$$

for almost every $\xi \in \mathbb{T}$ if $O_{\phi}$ is the outer function from the factorization of $f$. Equation (1.5) is due to the definition of inner functions, the Poisson integral formula, the harmonicity of $\log \left|O_{\phi}(z)\right|$ and the fact that

$$
\left|O_{\phi}(z)\right|=\exp \left(\int_{0}^{2 \pi} \log \phi\left(e^{i \theta}\right) d \mu_{z}(\theta)\right), \quad z \in \mathbb{D}
$$

The last inequality in (1.4) can be proved by applying Theorem 2. In the argument of the first inequality, the inner-outer factorization, the Schwarz-Pick lemma, and an upper estimate for $\left|O_{\phi}^{\prime}\right|$ from [6] are the main tools. It is worth underlining that Bøe's idea to make an upper estimate for $\left|f^{\prime}\right|$ by using the factorization seems to be quite effective. Another way to prove results like Theorem 3 is to use a modification of Theorem 2 together with the well-known equation

$$
\int_{0}^{2 \pi}\left|f\left(e^{i \theta}\right)-f(z)\right|^{2} d \mu_{z}(\theta)=\int_{0}^{2 \pi}\left|f\left(e^{i \theta}\right)\right|^{2} d \mu_{z}(\theta)-|f(z)|^{2}, \quad z \in \mathbb{D}
$$

but this method of Dyakonov has the obvious defect that it works only when $f \in H^{2}$. The advantage of this method in the case where $2 \leqslant p<\infty, 0<q<\infty, q / 2-1<$ $\alpha<q-1$, and $v(z)=(1-|z|)^{\alpha}$ is that $F_{1}(f)+F_{2}(f)$ in Theorem 3 can be replaced by

$$
\int_{0}^{1}\left(\int_{0}^{2 \pi}\left(\int_{0}^{2 \pi}\left|f\left(e^{i \theta}\right)\right|^{2} d \mu_{r e^{i t}}(\theta)-\left|f\left(r e^{i t}\right)\right|^{2}\right)^{p / 2} d t\right)^{q / p} \frac{v(r)}{(1-r)^{q}} d r
$$


see [9, Proposition 2.2(b)]. It is an open problem to prove a corresponding estimate for general weights.

Next we give an example that shows that the hypothesis $v \in \mathcal{D} \cap \widehat{\mathcal{D}}_{q}$ in Theorem 3 for $p \geqslant 2$ is sharp in a certain sense. Note that the example is a modification of [22, Example 8]. Before the statement we fix some notation. Write $f \lesssim g$ if there exists a constant $C>0$ such that $f \leqslant C g$, while $f \gtrsim g$ is understood analogously. If $f \lesssim g$ and $f \gtrsim g$, then we write $f \asymp g$.

Example 4 Let $2 \leqslant p<\infty, q=p, v(z)=(1-|z|)^{p-1}$, and $d A(z)$ be the twodimensional Lebesgue measure $d x d y$. Let $f$ be an inner function such that

$$
\int_{\{z \in \mathbb{D}:|f(z)|<\varepsilon\}} \frac{d A(z)}{1-|z|}=\infty
$$

for some $\varepsilon \in(0,1)$. The existence of such $f$ is guaranteed by [7, Theorem 5]. Then

$$
F_{1}(f)+F_{2}(f)=F_{1}(f) \asymp \int_{\mathbb{D}}(1-|f(z)|)^{p}(1-|z|)^{-1} d A(z)=\infty,
$$

while

$$
\left\|f^{\prime}\right\|_{A_{v}^{p, q}}^{q}+\|f\|_{H^{p}}^{q}=\left\|f^{\prime}\right\|_{A_{p-1}^{p, p}}^{p}+1<\infty
$$

by the well-known inclusion

$$
H^{p} \subset\left\{g: g^{\prime} \in A_{p-1}^{p, p}\right\}, \quad 2 \leqslant p<\infty
$$

which originates from [13].

We close the section by explaining how the remainder of this paper is organized. Auxiliary results on weights are recalled in the next section. The utility of Theorem 3 is demonstrated in Sects. 3 and 4. More precisely, in Sect. 3, we prove the factorization that states that, for any $f \in \mathcal{B}_{v}^{p, q}$, there exist $f_{1}, f_{2} \in \mathcal{B}_{v}^{p, q} \cap H^{\infty}$ such that $f=f_{1} / f_{2}$. Section 4 begins with a result giving a sufficient and necessary condition guaranteeing that the product of $f \in H^{p}$ and an inner function belongs to $\mathcal{B}_{\nu}^{p, q}$. As a consequence of this theorem, we obtain some results on zero sets of $\mathcal{B}_{v}^{p, p}$. Sections 5,6 , and 7 consist of the proofs of Theorems 1,2, and 3, respectively.

\section{Auxiliary Results on Weights}

In this section, we recall some basic properties of weights in $\widehat{\mathcal{D}}$ and $\check{\mathcal{D}}$. These properties are needed in subsequent sections. Another reason for these results is to help the reader to understand the nature of weights in $\mathcal{D}$. We begin with a result that is essentially [19, Lemma 3]; see also [18, Lemma 2.1].

Lemma A Let $v$ be a weight. Then the following statements are equivalent: 
(i) $v \in \widehat{\mathcal{D}}$.

(ii) There exist $C=C(v)>0$ and $\beta=\beta(v)>0$ such that

$$
\widehat{v}(r) \leqslant C\left(\frac{1-r}{1-s}\right)^{\beta} \widehat{v}(s), \quad 0 \leqslant r \leqslant s<1 .
$$

(iii) There exist $C=C(\nu)>0$ and $\gamma=\gamma(\nu)>0$ such that

$$
\int_{0}^{r}\left(\frac{1-r}{1-s}\right)^{\gamma} v(s) d s \leqslant C \widehat{v}(r), \quad 0 \leqslant r<1 .
$$

(iv) The estimate

$$
\int_{0}^{1} s^{x} v(s) d s \asymp \widehat{v}\left(1-\frac{1}{x}\right), \quad 1 \leqslant x<\infty,
$$

is satisfied.

From the point view of our main results, Lemma A(iii) is interesting because it states that $v \in \widehat{\mathcal{D}}$ if and only if $v \in \widehat{\mathcal{D}}_{p}$ for some $p>0$. This means that $\widehat{\mathcal{D}}=$ $\bigcup_{p>0} \widehat{\mathcal{D}}_{p}$. Nevertheless, Lemma A(ii) gives maybe the most interesting description for $\widehat{\mathcal{D}}$. Together with its $\check{\mathcal{D}}$ counterpart below it offers a very practical characterization for weights in $\mathcal{D}$. Essentially this characterization says that $\widehat{v}$ is normal in the sense of A. L. Shields and D. L. Williams [25].

Lemma B Let $v$ be a weight. Then $v \in \check{\mathcal{D}}$ if and only if there exist $C=C(v)>0$ and $\alpha=\alpha(v)>0$ such that

$$
\widehat{v}(s) \leqslant C\left(\frac{1-s}{1-r}\right)^{\alpha} \widehat{v}(r), \quad 0 \leqslant r \leqslant s<1 .
$$

Lemma B originates from [20], and it can be proved in a corresponding manner as Lemma A(ii). See in particular the proof of [18, Lemma 2.1].

By the definition of class $\widehat{\mathcal{D}}_{p}$, it is clear that $\widehat{\mathcal{D}}_{p} \subset \widehat{\mathcal{D}}_{p+\varepsilon}$ for any $\varepsilon>0$. Next we state [21, Lemma 3], which shows that also the converse inclusion is true for sufficiently small $\varepsilon=\varepsilon(\nu, p)>0$. The proof of this result is based on integration by parts. Note that $\widehat{\mathcal{D}}_{p}(v)$ in the statement is defined by (1.1).

Lemma C If $0<p<\infty$ and $v \in \widehat{\mathcal{D}}_{p}$, then $v \in \widehat{\mathcal{D}}_{p-\varepsilon}$ for any $\varepsilon \in\left(0, \frac{p}{\widehat{\mathcal{D}}_{p}(v)+1}\right)$.

The last result of this section is [22, Lemma 5], which shows that $v \in \mathcal{D}$ in the norm $\|f\|_{A_{v}^{p, q}}$ can be replaced by $\widehat{v}(z) /(1-|z|)$ without losing any essential information.

Lemma $\mathrm{D}$ Let $0<p, q<\infty$ and $v$ be a weight. 
(i) If $v \in \check{\mathcal{D}}$, then there exists $C=C(v)>0$ such that

$$
\|f\|_{A_{v}^{p, q}}^{q} \geqslant C \int_{0}^{1} M_{p}^{q}(r, f) \frac{\widehat{v}(r)}{1-r} d r, \quad f \in \mathcal{H}(\mathbb{D}) .
$$

(ii) If $v \in \widehat{\mathcal{D}}$, then there exists $C=C(v)>0$ such that

$$
\|f\|_{A_{v}^{p, q}}^{q} \leqslant C \int_{0}^{1} M_{p}^{q}(r, f) \frac{\widehat{v}(r)}{1-r} d r, \quad f \in \mathcal{H}(\mathbb{D}) .
$$

For $v \in \mathcal{D}$, Lemmas A(ii) and B yield

$$
\widehat{v}(r) \asymp \int_{r}^{1} \frac{\widehat{v}(s)}{1-s} d s, \quad 0 \leqslant r<1 .
$$

In [22], Lemma D is proved by applying this fact together with partial integrations. An alternative way to prove results like Lemma $\mathrm{D}$ is to split the integral with respect to $d r$ into infinitely many parts by using a dyadic partition, and then apply (2.1) together with the monotonicity of $M_{p}^{q}(r, f)$. An advantage of the last method is that $M_{p}^{q}(r, f)$ can be easily replaced by a certain monotonic function $g(r)$. This observation will be utilized several times in the argument of Theorem 2.

\section{Quotient Factorization}

Recall that if $f \in H^{p}$ for some $p>0$, then there exist $f_{1}, f_{2} \in H^{\infty}$ such that $f=$ $f_{1} / f_{2}$. This is an important consequence of classical factorization [8, Theorem 2.1] by F. and R. Nevanlinna. The main purpose of this section is to give the following $\mathcal{B}_{v}^{p, q}$ counterpart to the above-mentioned result.

Theorem 5 Let $1 \leqslant p<\infty, 0<q<\infty$, and $v \in \mathcal{D} \cap \widehat{\mathcal{D}}_{q}$. If $f \in \mathcal{B}_{v}^{p, q}$, then there exist $f_{1}, f_{2} \in \mathcal{B}_{v}^{p, q} \cap H^{\infty}$ such that $f=f_{1} / f_{2}$ and $f_{2}$ is an outer function.

It is worth mentioning that [16, Theorem 9.19] is a similar type of result as Theorem 5 with a different hypothesis for $v$. Moreover, we note that Theorem 5 generalizes [2, Corollary 2.7], [6, Theorem 3.4] and [9, Corollary 3.4]. For its argument we need an extension of [6, Theorem 3.3]. Note that a part of what follows is really inspired by [6].

Proposition 6 Let $1 \leqslant p<\infty, 0<q<\infty, v \in \mathcal{D} \cap \widehat{\mathcal{D}}_{q}$ and $f \in H^{p}$ be the product of an inner function $I$ and an outer function $O_{\phi}$. Then there exists a constant $C=C(p, q, v)>0$ such that

$$
\begin{aligned}
& \left\|O_{\max \{\phi, 1\}}^{\prime}\right\|_{A_{v}^{p, q}}^{q}+\left\|\left(I O_{\min \{\phi, 1\}}\right)^{\prime}\right\|_{A_{v}^{p, q}}^{q}+\left\|O_{\max \{\phi, 1\}}\right\|_{H^{p}}^{q} \\
& \quad \leqslant C\left(\left\|f^{\prime}\right\|_{A_{v}^{p, q}}^{q}+\|f\|_{H^{p}}^{q}+1\right) .
\end{aligned}
$$


Before the proof of Proposition 6, we note that the quantities $F_{1}(f)$ and $F_{2}(f)$ in Theorem 3 are used repeatedly hereafter.

Proof Let us begin by noting that $\left|O_{\phi}\left(e^{i \theta}\right)\right|=\phi\left(e^{i \theta}\right),\left|O_{\max \{\phi, 1\}}\left(e^{i \theta}\right)\right|=$ $\max \left\{\phi\left(e^{i \theta}\right), 1\right\}$, and

$$
\begin{aligned}
\max \left\{\phi\left(e^{i \theta}\right), 1\right\}-\phi\left(e^{i \theta}\right) & =\frac{\max \left\{\phi\left(e^{i \theta}\right), 1\right\}-\phi\left(e^{i \theta}\right)}{\max \left\{\phi\left(e^{i \theta}\right), 1\right\}} \\
& \leqslant\left|O_{\max \{\phi, 1\}}(z)\right|\left(1-\frac{\phi\left(e^{i \theta}\right)}{\max \left\{\phi\left(e^{i \theta}\right), 1\right\}}\right)
\end{aligned}
$$

for all $z \in \mathbb{D}$ and almost every $\theta \in[0,2 \pi)$. Using these facts together with Jensen's inequality [10, Chapter I, Lemma 6.1] and the definition of outer functions, we obtain

$$
\begin{aligned}
& \int_{0}^{2 \pi}\left|O_{\max \{\phi, 1\}}\left(e^{i \theta}\right)\right| d \mu_{z}(\theta)-\int_{0}^{2 \pi}\left|O_{\phi}\left(e^{i \theta}\right)\right| d \mu_{z}(\theta) \\
& \quad \leqslant\left|O_{\max \{\phi, 1\}}(z)\right|\left(1-\int_{0}^{2 \pi} \frac{\phi\left(e^{i \theta}\right)}{\max \left\{\phi\left(e^{i \theta}\right), 1\right\}} d \mu_{z}(\theta)\right) \\
& \quad=\left|O_{\max \{\phi, 1\}}(z)\right|\left(1-\int_{0}^{2 \pi} \exp \left(\log \phi\left(e^{i \theta}\right)-\log \max \left\{\phi\left(e^{i \theta}\right), 1\right\}\right) d \mu_{z}(\theta)\right) \\
& \quad \leqslant\left|O_{\max \{\phi, 1\}}(z)\right|\left(1-\frac{\left|O_{\phi}(z)\right|}{\left|O_{\max \{\phi, 1\}}(z)\right|}\right), \quad z \in \mathbb{D} .
\end{aligned}
$$

Consequently, the obvious inequality $|f(z)| \leqslant\left|O_{\phi}(z)\right|$ yields

$$
\begin{gathered}
\int_{0}^{2 \pi}\left|O_{\max \{\phi, 1\}}\left(e^{i \theta}\right)\right| d \mu_{z}(\theta)-\left|O_{\max \{\phi, 1\}}(z)\right| \\
\leqslant \int_{0}^{2 \pi}\left|f\left(e^{i \theta}\right)\right| d \mu_{z}(\theta)-|f(z)|, \quad z \in \mathbb{D} .
\end{gathered}
$$

Write $z=r e^{i t}$. Raising both sides of (3.1) to power $p$, integrating from 0 to $2 \pi$ with respect to $d t$, then raising both sides to power $q / p$, and finally integrating from 0 to 1 with respect to $v(r) d r /(1-r)^{q}$, we obtain $F_{1}\left(O_{\max \{\phi, 1\}}\right) \leqslant F_{1}(f)$.

Next we show $F_{2}\left(O_{\max \{\phi, 1\}}\right) \leqslant F_{2}(f)$. Set

$$
\Gamma_{1}=\Gamma_{1}(z, \phi)=\left\{\theta \in[0,2 \pi): \int_{0}^{2 \pi} \max \left\{\phi\left(e^{i s}\right), 1\right\} d \mu_{z}(s) \leqslant \phi\left(e^{i \theta}\right)\right\}
$$

and

$$
\Gamma_{2}=\Gamma_{2}(z, \phi)=\left\{\theta \in[0,2 \pi): \int_{0}^{2 \pi} \phi\left(e^{i s}\right) d \mu_{z}(s) \leqslant \phi\left(e^{i \theta}\right)\right\}, \quad z \in \mathbb{D} .
$$


Then elementary calculations yield

$$
\begin{aligned}
\int_{0}^{2 \pi}\left|\max \left\{\phi\left(e^{i \theta}\right), 1\right\}-\int_{0}^{2 \pi} \max \left\{\phi\left(e^{i s}\right), 1\right\} d \mu_{z}(s)\right| d \mu_{z}(\theta) \\
=2 \int_{\Gamma_{1}}\left(\max \left\{\phi\left(e^{i \theta}\right), 1\right\}-\int_{0}^{2 \pi} \max \left\{\phi\left(e^{i s}\right), 1\right\} d \mu_{z}(s)\right) d \mu_{z}(\theta) \\
\quad+\int_{0}^{2 \pi}\left(\int_{0}^{2 \pi} \max \left\{\phi\left(e^{i s}\right), 1\right\} d \mu_{z}(s)-\max \left\{\phi\left(e^{i \theta}\right), 1\right\}\right) d \mu_{z}(\theta) \\
=2 \int_{\Gamma_{1}}\left(\phi\left(e^{i \theta}\right)-\int_{0}^{2 \pi} \max \left\{\phi\left(e^{i s}\right), 1\right\} d \mu_{z}(s)\right) d \mu_{z}(\theta) \\
\leqslant 2 \int_{\Gamma_{2}}\left(\phi\left(e^{i \theta}\right)-\int_{0}^{2 \pi} \phi\left(e^{i s}\right) d \mu_{z}(s)\right) d \mu_{z}(\theta) \\
=\int_{0}^{2 \pi}\left|\phi\left(e^{i \theta}\right)-\int_{0}^{2 \pi} \phi\left(e^{i s}\right) d \mu_{z}(s)\right| d \mu_{z}(\theta), \quad z \in \mathbb{D} .
\end{aligned}
$$

Consequently, we obtain $F_{2}\left(O_{\max \{\phi, 1\}}\right) \leqslant F_{2}\left(O_{\phi}\right)=F_{2}(f)$ by doing a similar integral procedure as above. Now Theorem 3 together with the inequalities for $F_{1}(f)$ and $F_{2}(f)$ gives

$$
\left\|O_{\max \{\phi, 1\}}^{\prime}\right\|_{A_{v}^{p, q}}^{q}+\left\|O_{\max \{\phi, 1\}}\right\|_{H^{p}}^{q} \lesssim\left\|f^{\prime}\right\|_{A_{v}^{p, q}}^{q}+\|f\|_{H^{p}}^{q}+1 .
$$

By (3.3) it suffices to show

$$
\left\|\left(I O_{\min \{\phi, 1\}}\right)^{\prime}\right\|_{A_{v}^{p, q}}^{q} \lesssim\left\|f^{\prime}\right\|_{A_{v}^{p, q}}^{q}+\|f\|_{H^{p}}^{q} .
$$

Since

$$
\phi\left(e^{i \theta}\right)-\min \left\{\phi\left(e^{i \theta}\right), 1\right\} \geqslant\left|O_{\min \{\phi, 1\}}(z)\right|\left(\frac{\phi\left(e^{i \theta}\right)}{\min \left\{\phi\left(e^{i \theta}\right), 1\right\}}-1\right),
$$

we obtain

$$
\begin{aligned}
& \int_{0}^{2 \pi}\left|O_{\phi}\left(e^{i \theta}\right)\right| d \mu_{z}(\theta)-\left|O_{\phi}(z)\right| \\
& \quad \geqslant \int_{0}^{2 \pi}\left|O_{\min \{\phi, 1\}}\left(e^{i \theta}\right)\right| d \mu_{z}(\theta)-\left|O_{\min \{\phi, 1\}}(z)\right|, \quad z \in \mathbb{D},
\end{aligned}
$$


by arguing as above using Jensen's inequality. It follows that

$$
\begin{aligned}
\int_{0}^{2 \pi} & \left|f\left(e^{i \theta}\right)\right| d \mu_{z}(\theta)-|f(z)| \\
& =\left(\int_{0}^{2 \pi}\left|O_{\phi}\left(e^{i \theta}\right)\right| d \mu_{z}(\theta)-\left|O_{\phi}(z)\right|\right)+\left|O_{\phi}(z)\right|(1-|I(z)|) \\
& \geqslant\left(\int_{0}^{2 \pi}\left|O_{\min \{\phi, 1\}}\left(e^{i \theta}\right)\right| d \mu_{z}(\theta)-\left|O_{\min \{\phi, 1\}}(z)\right|\right)+\left|O_{\min \{\phi, 1\}}(z)\right|(1-|I(z)|) \\
& =\int_{0}^{2 \pi}\left|I O_{\min \{\phi, 1\}}\left(e^{i \theta}\right)\right| d \mu_{z}(\theta)-\left|I O_{\min \{\phi, 1\}}(z)\right|, \quad z \in \mathbb{D} .
\end{aligned}
$$

Hence it is easy to deduce $F_{1}\left(I O_{\min \{\phi, 1\}}\right) \leqslant F_{1}(f)$. Since

$$
F_{2}\left(I O_{\min \{\phi, 1\}}\right)=F_{2}\left(O_{\min \{\phi, 1\}}\right) \leqslant F_{2}\left(O_{\phi}\right)=F_{2}(f)
$$

can be shown by using a modification of (3.2), the desired estimate (3.4) follows from Theorem 3. This completes the proof.

Now we can easily prove Theorem 5 by using Proposition 6 .

Proof of Theorem 5 By the inner-outer factorization, there exist an inner function $I$ and an outer function $O_{\phi}$ such that $f=I O_{\phi}$. Since $O_{\phi}=O_{\min \{\phi, 1\}} O_{\max \{\phi, 1\}}$, we have $f=f_{1} / f_{2}$, where $f_{1}=I O_{\min \{\phi, 1\}}$ and $f_{2}=1 / O_{\max \{\phi, 1\}}$. Applying Proposition 6 together with the inequalities

$$
\left|O_{\min \{\phi, 1\}}(z)\right| \leqslant 1 \leqslant\left|O_{\max \{\phi, 1\}}(z)\right|
$$

and

$$
\left|f_{2}^{\prime}(z)\right| \leqslant\left|O_{\max \{\phi, 1\}}(z)\right|^{2}\left|f_{2}^{\prime}(z)\right|=\left|O_{\max \{\phi, 1\}}^{\prime}(z)\right|, \quad z \in \mathbb{D},
$$

we can check that $f_{1}$ and $f_{2}$ belong to $\mathcal{B}_{v}^{p, q} \cap H^{\infty}$. Moreover, it is obvious that $f_{2}$ is an outer function. Hence the proof is complete.

\section{Product of $f \in H^{p}$ and an Inner Function in $\mathcal{B}_{v}^{p, q}$}

Theorem 7 below gives a sufficient and necessary condition guaranteeing that the product of $f \in H^{p}$ and an inner function belongs to $\mathcal{B}_{v}^{p, q}$. This result generalizes [6, Corollary 3.2], the essential contents of [6, Corollary 3.1] and [9, Theorem 3.2].

Theorem 7 Let $1 \leqslant p<\infty, 0<q<\infty, v \in \mathcal{D} \cap \widehat{\mathcal{D}}_{q}, f \in H^{p}$, and I be an inner function. Then $f I \in \mathcal{B}_{v}^{p, q}$ if and only if $f \in \mathcal{B}_{v}^{p, q}$ and

$$
\int_{0}^{1}\left(\int_{0}^{2 \pi}\left(\frac{\left|f\left(r e^{i t}\right)\right|\left(1-\left|I\left(r e^{i t}\right)\right|\right)}{1-r}\right)^{p} d t\right)^{q / p} v(r) d r<\infty .
$$


Proof We have

$$
\begin{aligned}
\int_{0}^{2 \pi}\left|f I\left(e^{i \theta}\right)\right| d \mu_{z}(\theta)-|f I(z)|= & \left(\int_{0}^{2 \pi}\left|f\left(e^{i \theta}\right)\right| d \mu_{z}(\theta)-|f(z)|\right) \\
& +|f(z)|(1-|I(z)|)
\end{aligned}
$$

for all $z \in \mathbb{D}$. Write $z=r e^{i t}$. Raising both sides of (4.1) to power $p$, integrating from 0 to $2 \pi$ with respect to $d t$, then raising both sides to power $q / p$, integrating from 0 to 1 with respect to $v(r) d r /(1-r)^{q}$ and finally splitting the right-hand side into two parts by using well-known inequalities, we obtain

$$
F_{1}(f I) \asymp F_{1}(f)+\int_{0}^{1}\left(\int_{0}^{2 \pi}\left(\frac{\left|f\left(r e^{i t}\right)\right|\left(1-\left|I\left(r e^{i t}\right)\right|\right)}{1-r}\right)^{p} d t\right)^{q / p} v(r) d r .
$$

Since

$$
F_{2}(f I)+\|f I\|_{H^{p}}^{q}=F_{2}(f)+\|f\|_{H^{p}}^{q},
$$

the assertion follows from Theorem 3.

Recall that a subspace $X$ of $H^{p}$ satisfies the $F$-property if the hypothesis $f I \in X$, where $f \in H^{p}$ and $I$ is an inner function, implies $f \in X$. The $F$-property for $\mathcal{B}_{v}^{p, q}$ is a direct consequence of Theorem 7 . However, it is worth mentioning that if one just aims to prove the $F$-property for $\mathcal{B}_{v}^{p, q}$, our argument is maybe not the simplest one, taking into account the length of proofs of Theorem 3 and its auxiliary results. Ideas for an alternative proof can be found, for instance, in [16, Section 5.8.3].

A sequence $\left\{z_{n}\right\} \subset \mathbb{D}$ is said to be a zero set of $\mathcal{B}_{v}^{p, q}$ if there exists $f \in \mathcal{B}_{v}^{p, q}$ such that $\{z: f(z)=0\}=\left\{z_{n}\right\}$. Here each zero $z_{n}$ is repeated according to its multiplicity and function $f$ is not identically zero. Applying Theorem 7, we make some observations on zero sets of $\mathcal{B}_{v}^{p, p}$. More precisely, we concentrate on the case where $\left\{z_{n}\right\}$ is separated, which means that there exists $\delta=\delta\left(\left\{z_{n}\right\}\right)>0$ such that $d\left(z_{n}, z_{k}\right)>\delta$ for all $n \neq k$, where

$$
d(z, w)=\left|\frac{z-w}{1-\bar{z} w}\right|, \quad z, w \in \mathbb{D}
$$

is the pseudo-hyperbolic distance between points $z$ and $w$. Before these results some basic properties of Hardy spaces are recalled.

For $\left\{z_{n}\right\} \subset \mathbb{D}$ satisfying the Blaschke condition $\sum_{n}\left(1-\left|z_{n}\right|\right)<\infty$ and a point $\theta \in[0,2 \pi)$, the Blaschke product with zeros $\left\{z_{n}\right\}$ is defined by

$$
B(z)=e^{i \theta} \prod_{n} \frac{\left|z_{n}\right|}{z_{n}} \frac{z_{n}-z}{1-\bar{z}_{n} z}, \quad z \in \mathbb{D} .
$$

For $z_{n}=0$, the interpretation $\left|z_{n}\right| / z_{n}=-1$ is used. By factorization [8, Theorem 2.5] made by $\mathrm{F}$. Riesz, we know that any $f \in H^{p}$ for some fixed $p \in(0, \infty]$ can be 
represented in the form $f=B g$, where $B$ is a Blaschke product and $g \in H^{p}$ does not vanish in $\mathbb{D}$. More precisely, Beurling factorization [8, Theorem 2.8] asserts that $g$ is the product of an outer function and a singular inner function

$$
S(z)=\exp \left(\int_{\mathbb{T}} \frac{z+\xi}{z-\xi} d \sigma(\xi)+i \theta\right), \quad z \in \mathbb{D}
$$

where $\theta \in[0,2 \pi)$ is a constant and $\sigma$ is a positive measure on $\mathbb{T}$, singular with respect to the Lebesgue measure. Consequently, every zero set of $\mathcal{B}_{v}^{p, q}$ satisfies the Blaschke condition. With these preparations we are ready to state and prove the following result.

Corollary 8 Let $1 \leqslant p<\infty, v \in \mathcal{D} \cap \widehat{\mathcal{D}}_{p}$, and assume that $\left\{z_{n}\right\}$ is a finite union of separated sequences and zero set of $\mathcal{B}_{v}^{p, p}$. Then there exists an outer function $O_{\phi} \in \mathcal{B}_{v}^{p, p}$ such that

$$
\sum_{n}\left|O_{\phi}\left(z_{n}\right)\right|^{p} \frac{\widehat{v}\left(z_{n}\right)}{\left(1-\left|z_{n}\right|\right)^{p-1}}<\infty .
$$

Proof Let $\left\{z_{n}\right\}=\bigcup_{j=1}^{M}\left\{z_{n}^{j}\right\}$, where $M \in \mathbb{N}$ and each $\left\{z_{n}^{j}\right\}$ is separated. Let $B$ be the Blaschke product with zeros $\left\{z_{n}\right\}, S$ a singular inner function and $O_{\phi}$ an outer function such that $B S O_{\phi} \in \mathcal{B}_{v}^{p, q}$. By Theorem 7, we know that $O_{\phi}$ and $B O_{\phi}$ belong to $\mathcal{B}_{v}^{p, p}$. For $w \in \mathbb{D}$ and $0<r<1$, set

$$
\Delta(w, r)=\{z: d(z, w)<r\} \text { and } \Lambda(w, r)=\{z:|w-z|<r(1-|w|)\} .
$$

Since each $\left\{z_{n}^{j}\right\}$ is separated, we find $R_{j}, \delta_{j} \in(0,1)$ such that, for a fixed $j$, discs $\Lambda\left(z_{n}^{j}, R_{j}\right)$ are pairwise disjoint and the inclusion $\Delta\left(z_{n}^{j}, \delta_{j}\right) \subset \Lambda\left(z_{n}^{j}, R_{j}\right)$ is valid for every $n$. Hence $\widehat{v}$ is essentially constant in each disc $\Delta\left(z_{n}^{j}, \delta_{j}\right)$ by Lemma A(ii). Moreover,

$$
|B(z)| \leqslant\left|\frac{z_{n}^{j}-z}{1-\bar{z}_{n}^{j} z}\right| \leqslant \delta_{j}, \quad z \in \Delta\left(z_{n}^{j}, \delta_{j}\right) .
$$

Using these facts together with the subharmonicity of $\left|O_{\phi}\right|^{p}$, we obtain

$$
\begin{aligned}
& \sum_{n}\left|O_{\phi}\left(z_{n}\right)\right|^{p} \frac{\widehat{v}\left(z_{n}\right)}{\left(1-\left|z_{n}\right|\right)^{p-1}}=\sum_{j=1}^{M} \sum_{n}\left|O_{\phi}\left(z_{n}^{j}\right)\right|^{p} \frac{\widehat{v}\left(z_{n}^{j}\right)}{\left(1-\left|z_{n}^{j}\right|\right)^{p-1}} \\
& \quad \sum_{j=1}^{M} \sum_{n} \int_{\Delta\left(z_{n}^{j}, \delta_{j}\right)}\left|O_{\phi}(z)\right|^{p} d A(z) \frac{\widehat{v}\left(z_{n}^{j}\right)}{\left(1-\left|z_{n}^{j}\right|\right)^{p+1}} \\
& \quad \sum_{j=1}^{M} \sum_{n} \int_{\Delta\left(z_{n}^{j}, \delta_{j}\right)}\left|O_{\phi}(z)\right|^{p} \frac{\widehat{v}(z)}{(1-|z|)^{p+1}} d A(z)
\end{aligned}
$$




$$
\begin{aligned}
& \leqslant \sum_{j=1}^{M}\left(1-\delta_{j}\right)^{-p} \sum_{n} \int_{\Delta\left(z_{n}^{j}, \delta_{j}\right)}\left(\frac{\left|O_{\phi}(z)\right|(1-|B(z)|)}{1-|z|}\right)^{p} \frac{\widehat{v}(z)}{1-|z|} d A(z) \\
& \lesssim \int_{\mathbb{D}}\left(\frac{\left|O_{\phi}(z)\right|(1-|B(z)|)}{1-|z|}\right)^{p} \frac{\widehat{v}(z)}{1-|z|} d A(z)
\end{aligned}
$$

where $d A(z)$ is the two-dimensional Lebesgue measure. Now it suffices to show that the last integral in (4.2) is finite.

Set $\psi(z)=\widehat{v}(z) /(1-|z|)$ for $z \in \mathbb{D}$. Note that $\widehat{v}(r) \asymp \widehat{\psi}(r)$ for $0 \leqslant r<1$ by Lemmas A(ii) and B. Moreover, integrating by parts, one can show that $\nu \in \widehat{\mathcal{D}}_{p}$ if and only if

$$
\frac{(1-r)^{p}}{\widehat{v}(r)} \int_{0}^{r} \frac{\widehat{v}(s)}{(1-s)^{p+1}} d s \asymp 1, \quad r \rightarrow 1^{-} .
$$

In particular, $\psi \in \mathcal{D} \cap \widehat{\mathcal{D}}_{p}$ by the hypotheses of $\nu$. Since Lemma $\mathrm{D}$ implies $B O_{\phi}$ in $\mathcal{B}_{\psi}^{p, p}$, Theorem 7 gives

$$
\int_{\mathbb{D}}\left(\frac{\left|O_{\phi}(z)\right|(1-|B(z)|)}{1-|z|}\right)^{p} \frac{\widehat{v}(z)}{1-|z|} d A(z)<\infty .
$$

This completes the proof.

Recall that a sequence $\left\{z_{n}\right\} \subset \mathbb{D}$ is said to be uniformly separated if

$$
\inf _{n \in \mathbb{N}} \prod_{k \neq n}\left|\frac{z_{k}-z_{n}}{1-\bar{z}_{k} z_{n}}\right|>0
$$

and a finite union of uniformly separated sequences is called a Carleson-Newman sequence. It is worth mentioning that any Carleson-Newman sequence is a finite union of separated sequences satisfying the Blaschke condition, but the converse statement is not true. For $1<p<\infty, p-2<\alpha<p-1$, and a Carleson-Newman sequence $\left\{z_{n}\right\}$, we can give a sufficient and necessary condition for $\left\{z_{n}\right\}$ to be a zero set of $\mathcal{B}_{\alpha}^{p, p}$. This is a straightforward consequence of Theorem 7, Corollary 8, and the reasoning made in paper [4] by N. Arcozzi, D. Blasi and J. Pau.

Corollary 9 Let $1<p<\infty, p-2<\alpha<p-1$, and $\left\{z_{n}\right\}$ be a Carleson-Newman sequence. Then $\left\{z_{n}\right\}$ is a zero set of $\mathcal{B}_{\alpha}^{p, p}$ if and only if there exists an outer function $O_{\phi} \in \mathcal{B}_{\alpha}^{p, p}$ such that

$$
\sum_{n}\left|O_{\phi}\left(z_{n}\right)\right|^{p}\left(1-\left|z_{n}\right|\right)^{\alpha+2-p}<\infty .
$$

Proof Let $B$ be the Blaschke product with zeros $\left\{z_{n}\right\}$ and $O_{\phi} \in \mathcal{B}_{\alpha}^{p, p}$ an outer function satisfying (4.4). Then [14, Theorem 3.5] together with some elementary calculations 
gives

$$
\begin{aligned}
& \int_{\mathbb{D}}\left(\frac{\left|O_{\phi}(z)\right|(1-|B(z)|)}{1-|z|}\right)^{p}(1-|z|)^{\alpha} d A(z) \\
& \quad \leqslant 2 \int_{\mathbb{D}}\left|O_{\phi}(z)\right|^{p} \sum_{n} \frac{1-\left|z_{n}\right|^{2}}{\left|1-\bar{z}_{n} z\right|^{2}}(1-|z|)^{\alpha+1-p} d A(z) \\
& \quad \lesssim \sum_{n} \int_{\mathbb{D}}\left|O_{\phi}\left(z_{n}\right)\right|^{p} \frac{1-\left|z_{n}\right|^{2}}{\left|1-\bar{z}_{n} z\right|^{2}}(1-|z|)^{\alpha+1-p} d A(z) \\
& \quad+\sum_{n} \int_{\mathbb{D}}\left|O_{\phi}(z)-O_{\phi}\left(z_{n}\right)\right|^{p} \frac{1-\left|z_{n}\right|^{2}}{\left|1-\bar{z}_{n} z\right|^{2}}(1-|z|)^{\alpha+1-p} d A(z) \\
& =: \mathcal{I}_{1}+\mathcal{I}_{2} .
\end{aligned}
$$

Following the reasoning in the proof of [4, Proposition 3.2], it is easy to check that $\mathcal{I}_{1}$ and $\mathcal{I}_{2}$ are finite. More precisely, estimating in a natural manner, one can show

$$
\mathcal{I}_{1} \lesssim \sum_{n}\left|O_{\phi}\left(z_{n}\right)\right|^{p}\left(1-\left|z_{n}\right|\right)^{\alpha+2-p}<\infty
$$

In the argument of $\mathcal{I}_{2} \lesssim\left\|O_{\phi}^{\prime}\right\|_{A_{\alpha}^{p, p}}^{p}<\infty,\left[5\right.$, Lemma 2.1] and the hypothesis that $\left\{z_{n}\right\}$ is a Carleson-Newman sequence play key roles.

Since $O_{\phi} \in \mathcal{B}_{\alpha}^{p, p}$ and the first integral in (4.5) is finite, $B O_{\phi}$ belongs to $\mathcal{B}_{\alpha}^{p, p}$ by Theorem 7. Consequently, the implication $\Leftarrow$ is valid. The converse implication is a direct consequence of Corollary 8. Hence the proof is complete.

It is an open problem to prove a $\mathcal{B}_{v}^{p, p}$ counterpart of Corollary 9. One could try to prove such a result, for instance, assuming $\nu \in \mathcal{D} \cap \widehat{\mathcal{D}}_{p}$ and

$$
\sup _{0 \leqslant r<1} \frac{(1-r)^{p-1}}{\widehat{v}(r)} \int_{r}^{1} \frac{v(s)}{(1-s)^{p-1}} d s<\infty .
$$

In this case, the implication $\Leftarrow$ is the problematic part. An idea to approach this problem is to follow the argument of [4, Proposition 3.2] and aim to apply therein [3, Theorem 3.1] instead of [5, Lemma 2.1]. The downside of this method is that it leads to laborious computations of Bekollé-Bonami weights.

Corollaries 8 and 9 are related to some main results in [15] by J. Pau and J. A. Peláez. In particular, the equivalence (i) $\Leftrightarrow$ (ii) in [15, Theorem 1] follows from Corollary 9 by setting $p=2$. Moreover, Corollary 8 shows that the implication (i) $\Rightarrow$ (ii) in [15, Theorem 1] is valid also if $\left\{z_{n}\right\}$ in the statement is a finite union of separated sequences. Applying the last observation, we can also replace a Carleson-Newman sequence in [15, Corollary 1] by a finite union of separated sequences: If $0<\alpha<1$, $\left\{z_{n}\right\}$ is a finite union of separated sequences and zero set of $\mathcal{B}_{\alpha}^{2,2}$, then

$$
\int_{0}^{2 \pi} \log \left(\sum_{n} \frac{\left(1-\left|z_{n}\right|\right)^{\alpha+1}}{\left|e^{i \theta}-z_{n}\right|^{2}}\right) d \theta<\infty .
$$


This result offers a practical way to construct Blaschke sequences that are not zero sets of $\mathcal{B}_{\alpha}^{2,2}$; see [15, Theorem 2] and its proof.

Note that (4.2) and (4.5) together with the estimates for $\mathcal{I}_{1}$ and $\mathcal{I}_{2}$ are valid also if outer function $O_{\phi}$ is replaced by an arbitrary $f \in H^{p}$. Using this observation and Theorem 7, we can rewrite Corollary 9 in the following form.

Corollary 10 Let $1<p<\infty, p-2<\alpha<p-1, f \in H^{p}$, and $B$ be a Blaschke product associated with a Carleson-Newman sequence $\left\{z_{n}\right\}$. Then $f B \in \mathcal{B}_{\alpha}^{p, p}$ if and only if $f \in \mathcal{B}_{\alpha}^{p, p}$ and

$$
\sum_{n}\left|f\left(z_{n}\right)\right|^{p}\left(1-\left|z_{n}\right|\right)^{\alpha+2-p}<\infty .
$$

Corollary 10 is a partial improvement of the main result in M. Jevtić's paper [11]. More precisely, this paper contains an extended counterpart of Corollary 10 (in the sense of $p$ and $q$ ) with the defect $f \equiv 1$. It is also worth mentioning that Corollary 10 is not valid if the Carleson-Newman sequence $\left\{z_{n}\right\}$ is replaced by an arbitrary Blaschke sequence. This can be shown by studying the case where $f \equiv 1$ and $B$ is a Blaschke product with zeros on the positive real axis. More precisely, the counter example follows from [23, Theorem 1], which asserts that all such Blaschke products belong to $\mathcal{B}_{\alpha}^{p, p}$ for $1 / 2<p<\infty$ and $p-3 / 2<\alpha<\infty$.

Theorem 7 for $f \equiv 1$ (or Theorem 3 for inner functions) also has the extended counterpart [22, Theorem 1].

Theorem $\mathrm{E}$ Let $0<p, q<\infty$, and $v \in \mathcal{D}$. Then $v \in \widehat{\mathcal{D}}_{q}$ if and only if

$$
\left\|I^{\prime}\right\|_{A_{v}^{p, q}}^{q} \asymp \int_{0}^{1}\left(\int_{0}^{2 \pi}\left(\frac{1-\left|I\left(r e^{i \theta}\right)\right|}{1-r}\right)^{p} d \theta\right)^{q / p} v(r) d r
$$

for all inner functions I. Here the comparison constants may depend only on $p, q$, and $v$.

Theorem E confirms that the hypothesis $v \in \widehat{\mathcal{D}}_{q}$ in Theorems 3 and 7 is sharp in a certain sense. Studying the argument of this result in [22], we can also deduce that the proof of Theorem 3 is more straightforward when $f$ is an inner function, and the statement is valid for all $0<p<\infty$. It is also worth mentioning that results like Theorem $E$ have turned out to be useful in the theory of inner functions. Several by-products of Theorem E can be found in [22,24].

\section{Proof of Theorem 1}

Before the proof of Theorem 1 we recall [22, Lemma 6], which is a modification of [1, Lemma 5]. 
Lemma $\mathrm{F}$ If $0<p \leqslant 1$ and $g:[0,1) \rightarrow[0, \infty)$ is measurable, then

$$
\left(\int_{r}^{1} g(s) d s\right)^{p} \leqslant 2 \int_{r}^{1} \sup _{0 \leqslant x \leqslant s} g(x)^{p}(1-s)^{p-1} d s
$$

for $0 \leqslant r<1$.

Proof of Theorem 1 Let $\frac{4}{5} \leqslant s<1$ and choose $n=n(s) \in \mathbb{N} \backslash\{1,2,3,4\}$ such that $1-\frac{1}{n} \leqslant s<1-\frac{1}{n+1}$. Set $f_{n}(z)=z^{n}$ for $z \in \mathbb{D}$. Since

$$
\begin{aligned}
\left|e^{i n(\theta+h)}-e^{i n \theta}\right|^{2} & =\left|1-e^{i n h}\right|^{2}=2(1-\cos (n h))=2 n^{2} h^{2} \sum_{k=1}^{\infty}(-1)^{k-1} \frac{(n h)^{2(k-1)}}{(2 k) !} \\
& \geqslant 2 n^{2} h^{2}\left(\frac{1}{2}-\frac{n^{2} h^{2}}{24}\right) \geqslant \frac{2}{3} n^{2} h^{2}, \quad 0<h<\frac{2}{n},
\end{aligned}
$$

we have

$$
\begin{aligned}
& \int_{1 / 2}^{1} \omega_{p}\left(1-r, f_{n}\right)^{q} \frac{v(r)}{(1-r)^{q}} d r \\
& \left.\quad \int_{1 / 2}^{1-2 / n}+\int_{1-2 / n}^{1}\right)_{0<h<1-r} \sup _{0<\left.e^{i n h}\right|^{q}} \frac{v(r)}{(1-r)^{q}} d r \\
& \quad \geqslant \int_{1 / 2}^{1-2 / n}\left|1-e^{i 2}\right|^{q} \frac{v(r)}{(1-r)^{q}} d r+\int_{1-2 / n}^{s}\left|1-e^{i n(1-r)}\right|^{q} \frac{v(r)}{(1-r)^{q}} d r \\
& \gtrsim \int_{1 / 2}^{1-2 / n} \frac{v(r)}{(1-r)^{q}} d r+\int_{1-2 / n}^{s}(n+1)^{q} v(r) d r \\
& \geqslant \int_{1 / 2}^{1-2 / n} \frac{v(r)}{(1-r)^{q}} d r+(1-s)^{-q} \int_{1-2 / n}^{s} v(r) d r \\
& \geqslant \int_{1 / 2}^{s} \frac{v(r)}{(1-r)^{q}} d r \asymp \int_{0}^{s} \frac{v(r)}{(1-r)^{q}} d r .
\end{aligned}
$$

Using the hypothesis $v \in \widehat{\mathcal{D}}$ together with Lemma A(iv)(ii) in a similar manner as in the proof of [21, Theorem 1], we obtain

$$
\begin{aligned}
\left\|f_{n}^{\prime}\right\|_{A_{v}^{p, q}}^{q} & \asymp n^{q} \int_{0}^{1} r^{q(n-1)+1} v(r) d r \asymp n^{q} \int_{1-\frac{1}{q(n-1)+1}}^{1} v(r) d r \\
& \asymp n^{q} \int_{1-\frac{1}{n+1}}^{1} v(r) d r \leqslant \frac{\widehat{v}(s)}{(1-s)^{q}} .
\end{aligned}
$$

Finally combining the estimates above and using the inequality

$$
\int_{0}^{t} \frac{v(r)}{(1-r)^{q}} d r \lesssim 1 \asymp \frac{\widehat{v}(t)}{(1-t)^{q}}, \quad 0<t<\frac{4}{5},
$$


we deduce that if $v \in \widehat{\mathcal{D}}$ and (1.2) is satisfied for all $f \in H^{p}$, then $v \in \widehat{\mathcal{D}}_{p}$. Hence it suffices to prove the converse statement.

Let $f \in H^{p}, 0 \leqslant \theta<2 \pi, \frac{1}{2}<r<1$, and $0<h<\frac{1}{2}$. Set $\rho=r-h$ and let $\Gamma$ be the contour that goes first rapidly from $r e^{i \theta}$ to $\rho e^{i \theta}$, then along the circle $\{z:|z|=\rho\}$ to $\rho e^{i(\theta+h)}$, and finally rapidly to $r e^{i(\theta+h)}$. Since

$$
f\left(r e^{i(\theta+h)}\right)-f\left(r e^{i \theta}\right)=\int_{\Gamma} f^{\prime}(z) d z
$$

we have

$$
\begin{aligned}
\left|f\left(r e^{i(\theta+h)}\right)-f\left(r e^{i \theta}\right)\right| \leqslant & \int_{\rho}^{r}\left|f^{\prime}\left(s e^{i \theta}\right)\right| d s+\int_{\theta}^{\theta+h}\left|f^{\prime}\left(\rho e^{i t}\right)\right| d t \\
& +\int_{\rho}^{r}\left|f^{\prime}\left(s e^{i(\theta+h)}\right)\right| d s .
\end{aligned}
$$

Consequently, the discrete and continuous forms of Minkowski's inequality, a change of variable, and Hardy's convexity theorem yield

$$
\begin{aligned}
& \left(\int_{0}^{2 \pi}\left|f\left(r e^{i(\theta+h)}\right)-f\left(r e^{i \theta}\right)\right|^{p} d \theta\right)^{1 / p} \leqslant\left(\int_{0}^{2 \pi}\left(\int_{\rho}^{r}\left|f^{\prime}\left(s e^{i \theta}\right)\right| d s\right)^{p} d \theta\right)^{1 / p} \\
& \quad+\left(\int_{0}^{2 \pi}\left(\int_{0}^{h}\left|f^{\prime}\left(\rho e^{i(x+\theta)}\right)\right| d x\right)^{p} d \theta\right)^{1 / p} \\
& \quad+\left(\int_{0}^{2 \pi}\left(\int_{\rho}^{r}\left|f^{\prime}\left(s e^{i(\theta+h)}\right)\right| d s\right)^{p} d \theta\right)^{1 / p} \\
& \leqslant 2 \int_{\rho}^{r} M_{p}\left(s, f^{\prime}\right) d s+h M_{p}\left(\rho, f^{\prime}\right) \leqslant 3 \int_{r-h}^{r} M_{p}\left(s, f^{\prime}\right) d s
\end{aligned}
$$

Note that the deduction above can be found, for instance, in the proof of [8, Theorem 5.4].

By raising both sides of (5.1) to power $q$, adding $\sup _{0<h<1-t}$, and then integrating from $1 / 2$ to $r$ with respect to $v(t) d t /(1-t)^{q}$, we obtain

$$
\begin{aligned}
& \int_{1 / 2}^{r} \sup _{0<h<1-t}\left(\int_{0}^{2 \pi}\left|f\left(r e^{i(\theta+h)}\right)-f\left(r e^{i \theta}\right)\right|^{p} d \theta\right)^{q / p} \frac{v(t)}{(1-t)^{q}} d t \\
& \quad \lesssim \int_{1 / 2}^{r}\left(\int_{r-(1-t)}^{r} M_{p}\left(s, f^{\prime}\right) d s\right)^{q} \frac{v(t)}{(1-t)^{q}} d t .
\end{aligned}
$$

Letting $r \rightarrow 1^{-}$and using the monotone and mean convergence theorems together with the hypothesis $f \in H^{p}$, we deduce

$$
\int_{1 / 2}^{1} \omega_{p}(1-t, f)^{q} \frac{v(t)}{(1-t)^{q}} d t \lesssim \int_{1 / 2}^{1}\left(\int_{t}^{1} M_{p}\left(s, f^{\prime}\right) d s\right)^{q} \frac{v(t)}{(1-t)^{q}} d t=: \mathcal{I} .
$$


Hence it suffices to show $\mathcal{I} \lesssim\left\|f^{\prime}\right\|_{A_{v}^{p, q}}^{q}$. Note that the argument of this estimate uses ideas from [22].

If $q \leqslant 1$, then Lemma F with the choice $g(s)=M_{p}\left(s, f^{\prime}\right)$, Hardy's convexity theorem, Fubini's theorem, the hypothesis $v \in \widehat{\mathcal{D}}_{q}$, and Lemma $\mathrm{D}$ give

$$
\begin{aligned}
\mathcal{I} & \lesssim \int_{0}^{1} \frac{v(t)}{(1-t)^{q}} \int_{t}^{1} \sup _{0 \leqslant x \leqslant s} M_{p}^{q}\left(x, f^{\prime}\right)(1-s)^{q-1} d s d t \\
& =\int_{0}^{1} \frac{v(t)}{(1-t)^{q}} \int_{t}^{1} M_{p}^{q}\left(s, f^{\prime}\right)(1-s)^{q-1} d s d t \\
& =\int_{0}^{1} M_{p}^{q}\left(s, f^{\prime}\right)(1-s)^{q-1} \int_{0}^{s} \frac{v(t)}{(1-t)^{q}} d t d s \\
& \lesssim \int_{0}^{1} M_{p}^{q}\left(s, f^{\prime}\right) \frac{\widehat{v}(s)}{1-s} d s \asymp\left\|f^{\prime}\right\|_{A_{v}^{p, q}}^{q}
\end{aligned}
$$

for all $f \in \mathcal{H}(\mathbb{D})$. Hence the assertion for $q \leqslant 1$ is proved. If $q>1,0<\varepsilon<$ $q /\left(\widehat{\mathcal{D}}_{q}(v)+1\right)$, and $h(s)=(1-s)^{\frac{q-1-\varepsilon}{q}}$, then Hölder's inequality and Fubini's theorem yield

$$
\begin{aligned}
\mathcal{I} & \lesssim \int_{0}^{1} \int_{t}^{1} M_{p}^{q}\left(s, f^{\prime}\right) h(s)^{q} d s\left(\int_{t}^{1} h(r)^{-\frac{q}{q-1}} d r\right)^{q-1} \frac{v(t)}{(1-t)^{q}} d t \\
& \asymp \int_{0}^{1} \frac{v(t)}{(1-t)^{q-\varepsilon}} \int_{t}^{1} M_{p}^{q}\left(s, f^{\prime}\right)(1-s)^{q-1-\varepsilon} d s d t \\
& =\int_{0}^{1} M_{p}^{q}\left(s, f^{\prime}\right)(1-s)^{q-1-\varepsilon} \int_{0}^{s} \frac{v(t)}{(1-t)^{q-\varepsilon}} d t d s
\end{aligned}
$$

for all $f \in \mathcal{H}(\mathbb{D})$. Since $v \in \widehat{\mathcal{D}}_{q-\varepsilon}$ by Lemma C, the assertion for $q>1$ follows from Lemma D. This completes the proof.

Since

$$
\int_{0}^{1 / 2} \omega_{p}(1-t, f)^{q} \frac{v(t)}{(1-t)^{q}} d t \leqslant 2^{2 q}\|f\|_{H^{p}}^{q} \int_{0}^{1 / 2} v(t) d t
$$

by Minkowski's inequality, Theorem 1 has the following consequence.

Corollary 11 Let $1 \leqslant p<\infty, 0<q<\infty$, and $v \in \mathcal{D} \cap \widehat{\mathcal{D}}_{q}$. Then there exists a constant $C=C(p, q, v)>0$ such that

$$
\int_{0}^{1} \omega_{p}(1-r, f)^{q} \frac{v(r)}{(1-r)^{q}} d r \leqslant C\left(\left\|f^{\prime}\right\|_{A_{v}^{p, q}}^{q}+\|f\|_{H^{p}}^{q}\right)
$$

for all $f \in H^{p}$.

Note that Corollary 11 is a part of Theorem 2. We state it here as an independent result because it is needed for the proof of Theorem 2 . 


\section{Proof of Theorem 2}

We go directly to the proof of Theorem 2 .

Proof of Theorem 2 Let $0 \leqslant r<1$ and $0 \leqslant t<2 \pi$. Since

$$
\int_{0}^{2 \pi} \frac{e^{i \theta} d \theta}{\left(e^{i \theta}-r e^{i t}\right)^{2}}=0
$$

Cauchy's integral formula gives

$$
\begin{aligned}
\left|f^{\prime}\left(r e^{i t}\right)\right| & =\frac{1}{2 \pi\left(1-r^{2}\right)}\left|\int_{0}^{2 \pi}\left(f\left(e^{i \theta}\right)-f\left(r e^{i t}\right)\right) \frac{e^{i \theta}\left(1-r^{2}\right)}{\left(e^{i \theta}-r e^{i t}\right)^{2}} d \theta\right| \\
& \leqslant \frac{1}{1-r} \int_{0}^{2 \pi}\left|f\left(e^{i \theta}\right)-f\left(r e^{i t}\right)\right| d \mu_{r e^{i t}}(\theta), \quad f \in H^{1} .
\end{aligned}
$$

Raising both sides to power $p$, integrating from 0 to $2 \pi$ with respect to $d t$, then raising both sides to power $q / p$, and finally integrating from 0 to 1 with respect to $v(r) d r$, we obtain

$$
\left\|f^{\prime}\right\|_{A_{v}^{p, q}}^{q} \leqslant \int_{0}^{1}\left(\int_{0}^{2 \pi}\left(\int_{0}^{2 \pi}\left|f\left(e^{i \theta}\right)-f\left(r e^{i t}\right)\right| d \mu_{r e^{i t}}(\theta)\right)^{p} d t\right)^{q / p} \frac{v(r)}{(1-r)^{q}} d r
$$

for all $f \in H^{p}$.

Let $f \in H^{p}, 0<q \leqslant 1$, and set

$$
\mathcal{I}(r)=\left(\int_{0}^{2 \pi}\left(\int_{0}^{2 \pi}\left|f\left(e^{i \theta}\right)-f\left(r e^{i t}\right)\right| d \mu_{r e^{i t}}(\theta)\right)^{p} d t\right)^{q / p} .
$$

By the proof of [9, Theorem 2.1], we know that

$$
\mathcal{I}(r) \lesssim\left(\sum_{k=0}^{\infty} 2^{-k} \omega_{p}\left(2^{k}(1-r), f\right)\right)^{q}
$$

Hence the sub-additivity of $g(x)=x^{q}$ for $x \geqslant 0$ and Fubini's theorem give

$$
\int_{0}^{1} \frac{\mathcal{I}(r) v(r)}{(1-r)^{q}} d r \lesssim \sum_{k=0}^{\infty} 2^{-q k} \int_{0}^{1} \omega_{p}\left(2^{k}(1-r), f\right)^{q} \frac{v(r)}{(1-r)^{q}} d r
$$

Next we show that the weight $v(r)$ in the right-hand side can be replaced by $\frac{\widehat{v}(r)}{1-r}$ without losing any essential information.

Set $\psi(z)=\widehat{v}(z) /(1-|z|)$ for $z \in \mathbb{D}$, and recall that $\widehat{v}(r) \asymp \widehat{\psi}(r)$ for $0 \leqslant r<1$ by Lemmas $\mathrm{A}(\mathrm{ii})$ and $\mathrm{B}$. In particular, $\psi$ belongs to class $\mathcal{D}$, and thus, there exist 
$K=K(\psi)>1$ and $C=C(\psi)>1$ such that

$$
\widehat{\psi}(r) \geqslant C \widehat{\psi}\left(1-\frac{1-r}{K}\right), \quad 0 \leqslant r<1 .
$$

Let $k \in \mathbb{N} \cup\{0\}$ and $r_{n}=1-K^{-n}$ for $n \in \mathbb{N} \cup\{0\}$. Using (6.4) together with Lemma A(ii), we obtain

$$
\begin{aligned}
(C-1) \widehat{\psi}\left(r_{n+1}\right) & =C \widehat{\psi}\left(1-\frac{1-r_{n}}{K}\right)-\widehat{\psi}\left(r_{n+1}\right) \leqslant \widehat{\psi}\left(r_{n}\right)-\widehat{\psi}\left(r_{n+1}\right) \\
& =\int_{r_{n}}^{r_{n+1}} \psi(r) d r \leqslant \widehat{\psi}\left(r_{n}\right) \asymp \widehat{\psi}\left(r_{n+1}\right) .
\end{aligned}
$$

Now Minkowski's inequality, the monotonicity of $\omega_{p}(s, f)$ with $s$ and (6.5) yield

$$
\begin{aligned}
\int_{0}^{1} \omega_{p}\left(2^{k}(1-r), f\right)^{q} \frac{v(r)}{(1-r)^{q}} d r \\
\quad \lesssim \sum_{n=1}^{\infty} \int_{r_{n}}^{r_{n+1}} \omega_{p}\left(2^{k}(1-r), f\right)^{q} \frac{v(r)}{(1-r)^{q}} d r+\|f\|_{H^{p}}^{q} \\
\leqslant \sum_{n=1}^{\infty} \omega_{p}\left(2^{k}\left(1-r_{n}\right), f\right)^{q} \frac{\widehat{v}\left(r_{n}\right)}{\left(1-r_{n+1}\right)^{q}}+\|f\|_{H^{p}}^{q} \\
\asymp \sum_{n=1}^{\infty} \omega_{p}\left(2^{k}\left(1-r_{n}\right), f\right)^{q} \frac{\widehat{\psi}\left(r_{n}\right)}{\left(1-r_{n}\right)^{q}}+\|f\|_{H^{p}}^{q} \\
\asymp \sum_{n=0}^{\infty} \omega_{p}\left(2^{k}\left(1-r_{n+1}\right), f\right)^{q} \frac{\int_{r_{n}}^{r_{n+1}} \psi(r) d r}{\left(1-r_{n}\right)^{q}}+\|f\|_{H^{p}}^{q} \\
\leqslant \sum_{n=0}^{\infty} \int_{r_{n}}^{r_{n+1}} \omega_{p}\left(2^{k}(1-r), f\right)^{q} \frac{\psi(r)}{(1-r)^{q}} d r+\|f\|_{H^{p}}^{q} \\
=\int_{0}^{1} \omega_{p}\left(2^{k}(1-r), f\right)^{q} \frac{\widehat{v}(r)}{(1-r)^{q+1}} d r+\|f\|_{H^{p}}^{q} .
\end{aligned}
$$

It is worth noting that a similar deduction works also in the opposite direction.

Using (6.3) and (6.6), we obtain

$$
\begin{aligned}
\int_{0}^{1} \frac{\mathcal{I}(r) v(r)}{(1-r)^{q}} d r \lesssim & {\left[\sum_{k=0}^{\infty} 2^{-q k} \int_{0}^{1-2^{-k}} \omega_{p}\left(2^{k}(1-r), f\right)^{q} \frac{\widehat{v}(r)}{(1-r)^{q+1}} d r+\|f\|_{H^{p}}^{q}\right] } \\
& +\sum_{k=0}^{\infty} 2^{-q k} \int_{1-2^{-k}}^{1} \omega_{p}\left(2^{k}(1-r), f\right)^{q} \frac{\widehat{v}(r)}{(1-r)^{q+1}} d r \\
= & : \mathcal{I}_{1}+\mathcal{I}_{2} .
\end{aligned}
$$


Minkowski's inequality, (4.3) with $p$ being replaced by $q$, and Lemma B yield

$$
\begin{aligned}
\mathcal{I}_{1} & \lesssim\|f\|_{H^{p}}^{q} \sum_{k=0}^{\infty} 2^{-q k} \int_{0}^{1-2^{-k}} \frac{\widehat{v}(r)}{(1-r)^{q+1}} d r \\
& \lesssim\|f\|_{H^{p}}^{q} \sum_{n=0}^{\infty} \widehat{v}\left(1-2^{-k}\right) \\
& \lesssim \widehat{v}(0)\|f\|_{H^{p}}^{q} \sum_{n=0}^{\infty} 2^{-\alpha k} \asymp\|f\|_{H^{p}}^{q}
\end{aligned}
$$

for some $\alpha=\alpha(v)>0$. The continuity of $\widehat{v}$, changes of variables, Fubini's theorem, and the hypothesis $v \in \mathcal{D}$ give

$$
\begin{aligned}
\mathcal{I}_{2} & \asymp \int_{0}^{1} \frac{\omega_{p}(1-s, f)^{q}}{(1-s)^{q+1}} \int_{0}^{\infty} \widehat{v}\left(1-2^{-k}(1-s)\right) d k d s \\
& =\frac{1}{\log 2} \int_{0}^{1} \frac{\omega_{p}(1-s, f)^{q}}{(1-s)^{q+1}} \int_{s}^{1} \frac{\widehat{v}(x)}{1-x} d x d s \\
& \asymp \int_{0}^{1} \omega_{p}(1-s, f)^{q} \frac{\widehat{v}(s)}{(1-s)^{q+1}} d s .
\end{aligned}
$$

Summarizing, we have shown

$$
\int_{0}^{1} \frac{\mathcal{I}(r) v(r)}{(1-r)^{q}} d r \lesssim \int_{0}^{1} \omega_{p}(1-s, f)^{q} \frac{\widehat{v}(s)}{(1-s)^{q+1}} d s+\|f\|_{H^{p}}^{q} .
$$

Applying a similar argument as in (6.6), we can replace $\widehat{v}(s)$ in the right-hand side of (6.7) by $v(s)(1-s)$. Consequently, (6.1) and Corollary 11 imply (1.3) for all $f \in H^{p}$. Hence the assertion for $q \leqslant 1$ is proved.

Let $1<q<\infty$. Then (6.2), the continuous form of Minkowski's inequality, (6.6), and well-known inequalities give

$$
\begin{aligned}
& \int_{0}^{1} \frac{\mathcal{I}(r) v(r)}{(1-r)^{q}} d r \lesssim \int_{0}^{1}\left(\sum_{k=0}^{\infty} 2^{-k} \omega_{p}\left(2^{k}(1-r), f\right)\right)^{q} \frac{v(r)}{(1-r)^{q}} d r \\
& \quad \leqslant\left(\sum_{k=0}^{\infty} 2^{-k}\left(\int_{0}^{1} \omega_{p}\left(2^{k}(1-r), f\right)^{q} \frac{v(r)}{(1-r)^{q}} d r\right)^{1 / q}\right)^{q} \\
& \lesssim\left(\sum_{k=0}^{\infty} 2^{-k}\left(\int_{0}^{1} \omega_{p}\left(2^{k}(1-r), f\right)^{q} \frac{\widehat{v}(r)}{(1-r)^{q+1}} d r\right)^{1 / q}\right)^{q}+\|f\|_{H^{p}}^{q} \\
& \asymp\left[\left(\sum_{k=0}^{\infty} 2^{-k}\left(\int_{0}^{1-2^{-k}} \omega_{p}\left(2^{k}(1-r), f\right)^{q} \frac{\widehat{v}(r)}{(1-r)^{q+1}} d r\right)^{1 / q}\right)^{q}+\|f\|_{H^{p}}^{q}\right]
\end{aligned}
$$




$$
\begin{aligned}
& +\left(\sum_{k=0}^{\infty} 2^{-k}\left(\int_{1-2^{-k}}^{1} \omega_{p}\left(2^{k}(1-r), f\right)^{q} \frac{\widehat{v}(r)}{(1-r)^{q+1}} d r\right)^{1 / q}\right)^{q} \\
= & : \mathcal{I}_{3}+\mathcal{I}_{4} .
\end{aligned}
$$

Minkowski's inequality, (4.3) with $p$ being replaced by $q$, and Lemma B yield

$$
\begin{aligned}
\mathcal{I}_{3} & \lesssim\|f\|_{H^{p}}^{q}\left(\sum_{k=0}^{\infty} 2^{-k}\left(\int_{0}^{1-2^{-k}} \frac{\widehat{v}(r)}{(1-r)^{q+1}} d r\right)^{1 / q}\right)^{q} \\
& \lesssim\|f\|_{H^{p}}^{q}\left(\sum_{k=0}^{\infty} \widehat{v}\left(1-2^{-k}\right)^{1 / q}\right)^{q} \asymp\|f\|_{H^{p}}^{q} .
\end{aligned}
$$

By Lemma B, there exists a constant $\alpha=\alpha(v)>0$ such that

$$
\widehat{v}\left(1-2^{-k}(1-s)\right) \lesssim 2^{-\alpha k} \widehat{v}(s), \quad 0 \leqslant s \leqslant 1-2^{-k}(1-s)<1 .
$$

Using this together with a change of variable and modification of (6.6), we get

$$
\begin{aligned}
\mathcal{I}_{4} & =\left(\sum_{k=0}^{\infty}\left(\int_{0}^{1} \omega_{p}\left(2^{k}(1-s), f\right)^{q} \frac{\widehat{v}\left(1-2^{-k}(1-s)\right)}{(1-s)^{q+1}} d s\right)^{1 / q}\right)^{q} \\
& \lesssim\left(\sum_{k=0}^{\infty} 2^{-\alpha k / q}\left(\int_{0}^{1} \omega_{p}(1-s, f)^{q} \frac{\widehat{v}(s)}{(1-s)^{q+1}} d s\right)^{1 / q}\right)^{q} \\
& \asymp \int_{0}^{1} \omega_{p}(1-s, f)^{q} \frac{\widehat{v}(s)}{(1-s)^{q+1}} d s \\
& \lesssim \int_{0}^{1} \omega_{p}(1-s, f)^{q} \frac{v(s)}{(1-s)^{q}} d s+\|f\|_{H^{p}}^{q} .
\end{aligned}
$$

Finally (6.1), (6.8), and Corollary 11 imply (1.3) for all $f \in H^{p}$. This completes the proof.

\section{Proof of Theorem 3}

Before the proof of Theorem 3 we recall the following result, which is a part of the argument of [6, Theorem 1.1].

Lemma G If $O_{\phi}$ is an outer function, then

$$
\begin{aligned}
& \left|O_{\phi}^{\prime}(z)\right| \leqslant \frac{4}{1-|z|}\left(\int_{0}^{2 \pi}\left|\phi\left(e^{i \theta}\right)-\int_{0}^{2 \pi} \phi\left(e^{i s}\right) d \mu_{z}(s)\right| d \mu_{z}(\theta)\right. \\
& \left.\quad+\int_{0}^{2 \pi} \phi\left(e^{i h}\right) d \mu_{z}(h)-\left|O_{\phi}(z)\right|\right)
\end{aligned}
$$


for all $z \in \mathbb{D}$.

Proof of Theorem 3 Let $f \in H^{p}$. Then there exist an inner function $I$ and an outer function $O_{\phi}$ such that $f=I O_{\phi}$. Hence the Schwarz-Pick lemma, Lemma G, and the fact that $\phi(\xi)=|f(\xi)|$ for almost every $\xi \in \mathbb{T}$ yield

$$
\begin{aligned}
& \left|f^{\prime}(z)\right|(1-|z|) \leqslant\left(\left|I(z) O_{\phi}^{\prime}(z)\right|+\left|I^{\prime}(z) O_{\phi}(z)\right|\right)(1-|z|) \\
& \leqslant\left|O_{\phi}^{\prime}(z)\right|(1-|z|)+2\left|O_{\phi}(z)\right|(1-|I(z)|) \\
& \quad \leqslant 4 \int_{0}^{2 \pi}|| f\left(e^{i \theta}\right)\left|-\int_{0}^{2 \pi}\right| f\left(e^{i s}\right)\left|d \mu_{z}(s)\right| d \mu_{z}(\theta) \\
& \quad+4\left(\int_{0}^{2 \pi}\left|f\left(e^{i h}\right)\right| d \mu_{z}(h)-|f(z)|\right)
\end{aligned}
$$

for all $z \in \mathbb{D}$. Write $z=r e^{i t}$. Raising both sides of (7.1) to power $p$, integrating from 0 to $2 \pi$ with respect to $d t$, then raising both sides to power $q / p$, integrating from 0 to 1 with respect to $v(r) d r /(1-r)^{q}$, and finally splitting the right-hand side into two parts, we obtain

$$
\left\|f^{\prime}\right\|_{A_{v}^{p, q}}^{q} \lesssim F_{1}(f)+F_{2}(f)
$$

which is the first inequality in (1.4).

Set

$$
\Gamma=\Gamma(z, f)=\left\{\theta \in[0,2 \pi): \int_{0}^{2 \pi}\left|f\left(e^{i s}\right)\right| d \mu_{z}(s) \leqslant\left|f\left(e^{i \theta}\right)\right|\right\}, \quad z \in \mathbb{D} .
$$

Then elementary calculations together with the subharmonicity of $|f|$ yield

$$
\begin{aligned}
& \int_{0}^{2 \pi}|| f\left(e^{i \theta}\right)\left|-\int_{0}^{2 \pi}\right| f\left(e^{i s}\right)\left|d \mu_{z}(s)\right| d \mu_{z}(\theta) \\
& \quad=2 \int_{\Gamma}\left(\left|f\left(e^{i \theta}\right)\right|-\int_{0}^{2 \pi}\left|f\left(e^{i s}\right)\right| d \mu_{z}(s)\right) d \mu_{z}(\theta) \\
& \quad \leqslant 2 \int_{\Gamma}\left(\left|f\left(e^{i \theta}\right)\right|-|f(z)|\right) d \mu_{z}(\theta), \quad z \in \mathbb{D} .
\end{aligned}
$$

It follows that

$$
\begin{aligned}
& \int_{0}^{2 \pi}|| f\left(e^{i \theta}\right)\left|-\int_{0}^{2 \pi}\right| f\left(e^{i s}\right)\left|d \mu_{z}(s)\right| d \mu_{z}(\theta)+\left(\int_{0}^{2 \pi}\left|f\left(e^{i h}\right)\right| d \mu_{z}(h)-|f(z)|\right) \\
& \quad \leqslant 2\left(\int_{\Gamma}+\int_{0}^{2 \pi}\right)\left|f\left(e^{i \theta}\right)-f(z)\right| d \mu_{z}(\theta) \\
& \quad \leqslant 4 \int_{0}^{2 \pi}\left|f\left(e^{i \theta}\right)-f(z)\right| d \mu_{z}(\theta), \quad z \in \mathbb{D} .
\end{aligned}
$$


Doing a corresponding integration procedure for this estimate as above and applying Theorem 2, we obtain

$$
F_{1}(f)+F_{2}(f) \lesssim\left\|f^{\prime}\right\|_{A_{v}^{p, q}}^{q}+\|f\|_{H^{p}}^{q},
$$

which is the last inequality in (1.4). This completes the proof.

Acknowledgements Open access funding is provided by University of Eastern Finland. The author thanks Antti Perälä and Toshiyuki Sugawa for valuable comments, Tohoku University for hospitality during his visit there, and the referees for careful reading of the manuscript.

Open Access This article is licensed under a Creative Commons Attribution 4.0 International License, which permits use, sharing, adaptation, distribution and reproduction in any medium or format, as long as you give appropriate credit to the original author(s) and the source, provide a link to the Creative Commons licence, and indicate if changes were made. The images or other third party material in this article are included in the article's Creative Commons licence, unless indicated otherwise in a credit line to the material. If material is not included in the article's Creative Commons licence and your intended use is not permitted by statutory regulation or exceeds the permitted use, you will need to obtain permission directly from the copyright holder. To view a copy of this licence, visit http://creativecommons.org/licenses/by/4.0/.

\section{References}

1. Ahern, P.: The Poisson integral of a singular measure. Can. J. Math. 35(4), 735-749 (1983)

2. Aleman, A.: Hilbert spaces of analytic functions between the Hardy and the Dirichlet space. Proc. Am. Math. Soc. 115(1), 97-104 (1992)

3. Aleman, A., Constantin, O.: Spectra of integration operators on weighted Bergman spaces. J. Anal. Math. 109, 199-231 (2009)

4. Arcozzi, N., Blasi, D., Pau, J.: Interpolating sequences on analytic Besov type spaces. Indiana Univ. Math. J. 58(3), 1281-1318 (2009)

5. Blasi, D., Pau, J.: A characterization of Besov-type spaces and applications to Hankel-type operators. Michigan Math. J. 56(2), 401-417 (2008)

6. Bøe, B.: A norm on the holomorphic Besov space. Proc. Am. Math. Soc. 131(1), 235-241 (2003)

7. Borichev, A.: Generalized Carleson-Newman inner functions. Math. Z. 275(3-4), 1197-1206 (2013)

8. Duren, P.: Theory of $H^{p}$ Spaces. Academic Press, New York (1970)

9. Dyakonov, K.M.: Besov spaces and outer functions. Michigan Math. J. 45(1), 143-157 (1998)

10. Garnett, J.: Bounded Analytic functions Revised, 1st edn. Springer, New York (2007)

11. Jevtić, M.: On Blaschke products in Besov spaces. J. Math. Anal. Appl. 149(1), 86-95 (1990)

12. Jevtić, M., Pavlović, M.: Besov-Lipschitz and mean Besov-Lipschitz spaces of holomorphic functions on the unit ball. Potential Anal. 38(4), 1187-1206 (2013)

13. Littlewood, J.E., Paley, R.E.A.C.: Theorems on Fourier series and power series (II). Proc. Lond. Math. Soc. (2) 42(1), 52-89 (1936)

14. Mashreghi, J.: Derivatives of inner functions, Fields Institute Monographs 31. Springer, New York; Fields Institute for Research in Mathematical Sciences, Toronto, ON (2013)

15. Pau, J., Peláez, J.A.: On the zeros of functions in Dirichlet-type spaces. Trans. Am. Math. Soc. 363(4), 1981-2002 (2011)

16. Pavlović, M.: Function Classes on the Unit Disc, An introduction, De Gruyter Studies in Mathematics, vol. 52. De Gruyter, Berlin (2013)

17. Pavlović, M.: On the moduli of continuity of $H^{p}$-functions with $0<p<1$. Proc. Edinburgh Math. Soc. (2) 35(1), 89-100 (1992)

18. Peláez, J. A.: Small weighted Bergman spaces, Proceedings of the Summer School in Complex and Harmonic Analysis, and Related Topics, Publ. Univ. East. Finl. Rep. Stud. For. Nat. Sci., vol. 22, Univ. East. Finl., Fac. Sci. For., Joensuu, pp. 29-98 (2016)

19. Peláez, J.A., Rättyä, J.: Embedding theorems for Bergman spaces via harmonic analysis. Math. Ann. 362(1-2), 205-239 (2015) 
20. Peláez, J. A., Rättyä, J.: Bergman projection induced by radial weight. Preprint available at https:// arxiv.org/pdf/1902.09837.pdf

21. Pérez-González, F., Rättyä, J.: Derivatives of inner functions in weighted Bergman spaces and the Schwarz-Pick lemma. Proc. Am. Math. Soc. 145(5), 2155-2166 (2017)

22. Reijonen, A.: Derivatives of inner functions in weighted mixed norm spaces. J. Geom. Anal. 29(3), 1859-1875 (2019)

23. Reijonen, A.: Derivatives of Blaschke products whose zeros lie in a Stolz domain and weighted Bergman spaces. Proc. Am. Math. Soc. 146(3), 1173-1180 (2018)

24. Reijonen, A., Sugawa, T.: Characterizations for inner functions in certain function spaces. Complex Anal. Oper. Theory 13(4), 1853-1871 (2019)

25. Shields, A.L., Williams, D.L.: Bounded projections, duality, and multipliers in spaces of analytic functions. Trans. Am. Math. Soc. 162, 287-302 (1971)

26. Stein, E.M.: Singular Integrals and Differentiability Properties of Functions. Princeton University Press, Princeton (1970)

Publisher's Note Springer Nature remains neutral with regard to jurisdictional claims in published maps and institutional affiliations. 